\title{
Eugenol, a Component of Holy Basil (Tulsi) and Common Spice Clove, Inhibits the Interaction Between SARS-CoV-2 Spike S1 and ACE2 to Induce Therapeutic Responses
}

\author{
Ramesh Kumar Paidi ${ }^{1} \cdot$ Malabendu Jana ${ }^{1} \cdot$ Sumita Raha ${ }^{1} \cdot$ Mary McKay ${ }^{1}$ Monica Sheinin ${ }^{1} \cdot$ Rama K. Mishra $^{2}$. \\ Kalipada Pahan ${ }^{1,3}$ (D)
}

Received: 25 August 2021 / Accepted: 6 October 2021 / Published online: 22 October 2021

(c) The Author(s), under exclusive licence to Springer Science+Business Media, LLC, part of Springer Nature 2021

\begin{abstract}
Spike S1 of Severe Acute Respiratory Syndrome Coronavirus 2 (SARS-CoV-2) binds to angiotensin-converting enzyme 2 (ACE2) on host cells to enter the cell and initiate COVID-19. Since ACE2 is a favorable enzyme, we were interested in finding a molecule capable of binding spike S1, but not ACE2, and inhibiting the interaction between spike S1 and ACE2. Holy basil (Tulsi) has a long history as a medicine for different human disorders. Therefore, we screened different components of Tulsi leaf and found that eugenol, but not other major components (e.g. ursolic acid, oleanolic acid and $\beta$-caryophylline), inhibited the interaction between spike S1 and ACE2 in an AlphaScreen-based assay. By in silico analysis and thermal shift assay, we also observed that eugenol associated with spike S1, but not ACE2. Accordingly, eugenol strongly suppressed the entry of pseudotyped SARS-CoV-2, but not vesicular stomatitis virus (VSV), into human ACE2-expressing HEK293 cells. Eugenol also reduced SARS-CoV-2 spike S1-induced activation of NF- $\kappa$ B and the expression of IL-6, IL-1 $\beta$ and TNFa in human A549 lung cells. Moreover, oral treatment with eugenol reduced lung inflammation, decreased fever, improved heart function, and enhanced locomotor activities in SARS-CoV-2 spike S1-intoxicated mice. Therefore, selective targeting of SARS-CoV-2 spike S1, but not ACE2, by eugenol may be beneficial for COVID-19 treatment.
\end{abstract}

Keywords COVID-19 $\cdot$ Eugenol $\cdot$ Spike S1 $\cdot$ ACE2 $\cdot$ Fever $\cdot$ Inflammation

\section{Introduction}

Holy basil or Tulsi (Ocimum tenuiflorum) is cultivated in Southeast Asia for religious and traditional medicine purposes (Cohen 2014). Tulsi is known to augment immunity that may help fight viral, bacterial and fungal infections. For example, in a 4 -week study in 24 healthy individuals, it has been found that supplementation of $300 \mathrm{mg}$ of holy basil extract is capable of increasing levels of IFN- $\gamma$, IL-4 and

Kalipada Pahan

Kalipada_Pahan@rush.edu

1 Department of Neurological Sciences, Rush University Medical Center, IL, Chicago, USA

2 Department of Biochemistry and Molecular Genetics, Feinberg School of Medicine, Northwestern University, Chicago, USA

3 Division of Research and Development, Jesse Brown Veterans Affairs Medical Center, Chicago, USA percentages of T-helper cells and natural killer cells (Mondal et al. 2011). These are the immune cells that are beneficial in protecting and defending the human body from viral infections. In addition, many cell culture and animals studies have delineated anti-inflammatory, antioxidant, anti-cancer, hepatoprotective, radioprotective, anxiolytic, adaptogenic, metabolic, and anti-diabetic effects of Tulsi leaf (Prakash and Gupta 2005; Baliga et al. 2013; Cohen 2014; Jamshidi and Cohen 2017).

The coronavirus disease 2019 (COVID-19) pandemic that started from the novel severe acute respiratory syndrome coronavirus 2 (SARS-CoV-2) in December 2019 is still continuing to kill thousands of people daily worldwide. To date, officially 4.8 million people lost their lives in the world from COVID-19. Although affected individuals manifest a wide array of symptoms, common symptoms of COVID-19 are fever, cough, and shortness of breath (Ledford 2020; Machhi et al. 2020). Severity to COVID-19 increases with age as well as preexisting conditions, such as hypertension, obesity, asthma, or diabetes. It 
has been found that severely ill COVID-19 patients suffer from cytokine storm, lung injury and multi-organ failure (Pia 2020). Although underlying mechanisms are poorly understood, COVID-19 is more lethal in men than it is in women (Mukherjee and Pahan 2021). While vaccination is underway and more than $50 \%$ people in USA are fully vaccinated, a specific and an effective antiviral and anti-inflammatory agent is also needed to treat this viral pandemic.

Angiotensin-converting enzyme 2 (ACE2) is a beneficial molecule as it converts angiotensin II (AngII), a vasoconstrictor, to Ang1-7, a vasodilator (Vickers et al. 2002; Zaman et al. 2002). Since the spike protein on the surface of SARSCoV-2 binds to ACE2 (Machhi et al. 2020; Stower 2020) to enter into human cells and the spike $\mathrm{S} 1$ subunit harbors the receptor-binding domain (RBD), we screened different components of Tulsi leaf and found that eugenol was capable of inhibiting the interaction between spike S1 and ACE2. In addition, eugenol inhibited the entry of pseudotyped SARSCoV-2, but not VSV, into human ACE2-expressing HEK293 cells and suppressed spike S1-induced activation of NF- $\kappa \mathrm{B}$ and expression of proinflammatory cytokines in human lungs cells. Oral administration of eugenol also decreased lung inflammation, reduced fever, inhibited arrhythmias, and enhanced locomotor activities in an animal model of COVID-19, indicating that naturally available eugenol may be beneficial for COVID-19.

\section{Materials and Methods}

\section{Reagents}

Eugenol, ursolic acid, oleanolic acid, and $\beta$-caryophylline were purchased from Sigma, St. Louis, MO. Methyl cellulose was bought from Spectrum Chemical, Gardena, CA. SARS-CoV-2 spike-pseudotyped lentiviral particles, VSV-G-pseudotyped particles and human ACE2-expressing HEK293 cells were purchased from GeneCopoeia, Rockville, MD. Recombinant COVID-19 Spike protein S1 (14-685) was purchased from Abeomics, San Diego, CA. Recombinant human ACE2 protein (18-739) was purchased from MyBiosource, San Diego, CA. Human A549 lung carcinoma cell line and F-12 K medium were obtained from ATCC, Manassas, VA. Hank's balanced salt solution, $0.05 \%$ trypsin, and antibiotic-antimycotic mixture were bought from Mediatech (Washington, DC). Fetal bovine serum (FBS) was obtained from Atlas Biologicals, Fort Collins, CO. ACE2:SARS-CoV-2 Spike Inhibitor Screening Assay Kit was purchased from BPS Bioscience, San Diego, CA. Mouse TNFa ELISA kit was bought from ThermoFisher, Waltham, MA.

\section{ACE2:SARS-CoV-2 Spike Binding Assay}

The efficacy of eugenol, ursolic acid, oleanolic acid, and $\beta$-caryophylline to dissociate the binding of SARSCoV-2 spike S1 with ACE2 was investigated using the ACE2:SARS-CoV-2 Spike inhibitor screening assay kit (BPS Bioscience, San Diego, CA) according to manufacturer's instructions as described by us recently (Paidi et al. 2021). Briefly, this is an AlphaScreen-based assay in which 96-well nickel-treated plate provided by the manufacturer was coated with ACE2 solution. After washing with immuno buffer and treatment with blocking buffer, different concentrations of eugenol, ursolic acid, oleanolic acid, and $\beta$-caryophylline were added to each well followed by addition of SARS-CoV-2 Spike (RBD)-Fc. After washing and incubation with blocking buffer, plates were treated with anti-mouse Fc-HRP followed by addition of an HRP substrate. Resultant chemiluminescence was monitored in a Perkin Elmer multimode microplate reader, Victor X5.

\section{In Silico Structural Analysis}

To study the interaction among eugenol, human ACE2 and SARS-CoV-2 spike S1, we performed in silico structural analysis was performed as described earlier (Roy et al. 2015; Rangasamy et al. 2018; Paidi et al. 2021). Briefly, by utilizing the protein preparation tools from the Schrodinger, Inc. platform, at first, we evaluated the quality of the crystal structure of human ACE2 and SARS-CoV-2 spike S1 followed by addition of hydrogens to the hydrogen bond orientation, charges, missing atoms, and side chains of the different residues of both the proteins. The 3D structure of eugenol was achieved from Zinc database. Finally, the complex structure was subjected to energy minimization in the Optimized Potential for Liquid Simulations (OPLS3) force field to make it torsion free. We applied the dynamic hydrogen bonding module for finding potential hydrogen bonds among the structures. We also evaluated other interactions such as hydrophobic interactions.

\section{Thermal Shift Assay}

Thermal shift assay was conducted using SYBR green realtime melting strategy using the thermal shift dye kit (Life Technologies) in Applied Biosystems 7500 standard real time thermal cycler. As described earlier (Roy et al. 2015, 2016; Patel et al. 2018), each reaction contained $250 \mathrm{ng}$ of either recombinant SARS-CoV-2 spike S1 or human ACE2 protein, $10 \mu \mathrm{M}$ eugenol, $10 \mu \mathrm{l}$ thermal shift buffer, and $2 \mu \mathrm{l}$ of dye. Thermal shift reaction was performed in dark. The 96-well PCR plate was loaded in the thermal cycler for the 
following two-stage program $\left(\left[25^{\circ} \mathrm{C}\right.\right.$ for $\left.2 \mathrm{~min}\right] 1$ cycle; $\left[27^{\circ} \mathrm{C}\right.$ for $15 \mathrm{~s}, 26^{\circ} \mathrm{C}$ for $\left.1 \mathrm{~min}\right] 70$ cycles; auto increment $1{ }^{\circ} \mathrm{C}$ for both stages). The filter was set at ROX with no quencher filter and no passive filter. While performing thermal shift assay with the combination of SARS-CoV-2 spike S1 and human ACE2, recombinant SARS-CoV-2 spike S1 and human ACE2 protein were mixed in equimolar amounts and incubated for $30 \mathrm{~min}$ at room temperature followed by addition of $10 \mu \mathrm{M}$ eugenol and reaction was carried out as described above. $\Delta \mathrm{Tm}$ was calculated in Graphpad Prism 8.4.3.

\section{Infection with Pseudotyped Lentiviral Particles}

Human ACE2-expressing HEK293 cells were plated in 24-well plates at $70-80 \%$ confluence followed by infection with lentiviral particles pseudotyped with either SARSCoV-2 spike S1 or VSV-G at an MOI of 0.5 as described (Cantuti-Castelvetri et al. 2020). These pseudoviruses (GeneCopoeia, Rockville, MD) contained Luciferase and green fluorescent protein (GFP) reporter genes that helped us to quantify virus entry into ACE2-expressing 293T cells just by monitoring either luciferase activity or GFP fluorescence. Cells were also infected with empty lentiviral (lentinaked) particles as control. After $48 \mathrm{~h}$ of infection, the entry of pseudovirus into HEK293 cells was checked by either luciferase activity or GFP fluorescence. Luciferase activity was assayed in total cell extracts using the Luciferase Assay System (Promega) in a TD-20/20 Luminometer (Turner Designs, Sunnyvale, CA) as described (Saha et al. 2006; Jana et al. 2013). GFP fluorescence was monitored in an Olympus BX-41 fluorescence microscope as described (Roy et al. 2013).

\section{Quantitative Real-time PCR Analysis}

Total RNA was isolated from mouse lungs and human A549 lung cells using Ultraspec-II RNA reagent (Biotecx Laboratories, Inc., Houston, TX), and RNAeasy Mini kit (Qiagen, Germantown, MD), respectively. To remove any contaminating genomic DNA, total RNA was digested with DNase. Real-time PCR was performed in the ABI-Prism7700 sequence detection system (Applied Biosystems, Foster City, CA) using the SYBR green real-time kit obtained from QuantaBio (Beverly, MA) as described before (Jana et al. 2008; Patel et al. 2019; Rangasamy et al. 2019). The following primer sequences were used:

TNF-a: Sense: 5'-TTCTGTCTACTGAACTTCGGGGTG ATCGGTCC- $3^{\prime}$.

Antisense: 5'-GTATGAGATAGCAAATCGGCTGAC GGTGTGGG-3'.

IL-1 $\beta$ : Sense: 5'-GGATATGGAGCAACAAGTGG-3'.
Antisense: 5'-ATGTACCAGTTGGGGAACT-3'. IL-6: Sense: 5'-GACAACTTTGGCATTGTGG-3'. Antisense: 5'-ATGCAGGGATGATGTTCTG-3'. GAPDH: Sense: 5'-GGTGAAGGTCGGTGTGAACG-3' . Antisense: 5'-TTGGCTCCACCCTTCAAGGTG-3'.

\section{Electrophoretic Mobility Shift Assay (EMSA)}

Nuclear extracts were prepared, and EMSA was performed as described previously (Pahan et al. 2001; Jana et al. 2013; Rangasamy et al. 2018) with minor modifications. We purchased IRDye infrared dye end-labeled oligonucleotides containing the consensus binding sequence for NF- $\kappa \mathrm{B}$ from Licor Biosciences. Nuclear extract $(6 \mu \mathrm{g})$ was incubated with binding buffer and with IRDye-labeled NF- $\kappa B$ probe for 20 min followed by separation on a $6 \%$ polyacrylamide gel in $0.25 \times$ TBE buffer (Tris borate-EDTA) and analysis by the Odyssey Infrared Imaging System (LI-COR Biosciences).

\section{Animals and Intoxication of C57/BL6 Mice with Recombinant SARS-CoV-2 Spike S1}

Mice were maintained and experiments conducted in accordance with National Institute of Health guidelines and were approved by the Rush University Medical Center IACUC. C57/BL6 mice (8-10 week old; Envigo) of both sexes were intoxicated with recombinant SARS-CoV-2 spike S1 (50 ng/ mouse/d) intranasally as described by us recently (Paidi et al. 2021). Briefly, recombinant spike S1 was dissolved in $2 \mu \mathrm{l}$ normal saline, mice were hold in supine position and $1 \mu \mathrm{l}$ volume was delivered into each nostril using a pipetman. Control mice received only $2 \mu \mathrm{l}$ saline.

\section{Eugenol Treatment}

Eugenol was mixed in $0.1 \%$ methylcellulose Starting from $5 \mathrm{~d}$ of SARS-CoV-2 spike S1 intoxication, C57/BL6 mice (8-10 week old; Envigo) of both sexes were treated orally with eugenol ( $25 \mathrm{mg} / \mathrm{kg}$ body weight/d) via gavage for $10 \mathrm{~d}$.

\section{Non-invasive ECG Recording}

ECG recording was performed as described by us recently (Paidi et al. 2021). Briefly, mice were acclimatized to the ECG pulse transducer pad (AD instruments TN 012/ST, USA) and the experimental housing conditions prior to ECG recording. ECG pulse transducer pad was placed around the heart of each animal and ECG recording was carried out for $120 \mathrm{~s}$. For ECG analysis, electrocardiography data were exported from the Labchart pro, version 8.0 (Power Lab 4/35 model) as raw data format and the digital signal processing was performed using this software. The recording was conducted for $120 \mathrm{~s}$ and the ECG signals were recorded at 
a sampling range of $20 \mathrm{mV}$ with 4 beats/s sampling rate and different ECG parameters were calculated as described (Paidi et al. 2021). The recording and analysis settings were kept same for all the experimental mice included in this study.

\section{ELISA for TNFa}

TNFa ELISA was performed in mouse serum as described earlier (Pahan et al. 1997; Saha et al. 2006; Mondal et al. 2020) using an assay kit (eBioscience) according to manufacturer's instruction.

\section{Statistical Analysis}

Statistical analyses were performed using GraphPad Prism 8.0 (GraphPad Software, Inc., La Jolla, CA). Mouse behavioral measures were examined by an independent one-way ANOVA using SPSS. Homogeneity of variance between test groups was examined using Levene's test. Post-hoc analyses were conducted using Tukey's tests. Other data were expressed as means $\pm \mathrm{SD}$ of three independent experiments. Statistical differences between means were calculated by the Student's $t$-test (two-tailed). A $p$-value of less than 0.05 $(p<0.05)$ was considered statistically significant.

\section{Results}

Eugenol Inhibits the Binding of SARS-CoV-2 Spike S1 with ACE2 The interaction of SARS-CoV-2 with its receptor ACE2 is critical for the infection of host cells and the pathogenesis of COVID-19. Therefore, we screened different molecules based on the inhibition of interaction between ACE2 and the receptor-binding domain (RBD) of SARS-CoV-2 spike S1 (Du et al. 2009). Since supplementation of leaves or leaf extracts of holy basil or Tulsi (Ocimum tenuiflorum) induces antiviral immunity (Mondal et al. 2011; Sahoo et al. 2016), we decided to screen major components (ursolic acid, oleanolic acid, eugenol, and $\beta$-caryophylline) of Tulsi leaf. As described recently (Paidi et al. 2021), we employed a chemiluminescence-based ACE2:SARS-CoV-2 spike S1 binding assay (catalog\# 79,936; BPS Bioscience). We noticed that SARS-CoV-2 spike S1 binding to immobilized ACE2 was inhibited by different doses of eugenol (Fig. 1A). However, ursolic acid, oleanolic acid and $\beta$-caryophylline remained unable to inhibit the association between SARSCoV-2 spike S1 and ACE2 (Fig. 1A), indicating the specificity of the effect.

In an effort to understand whether eugenol binds to SARS-CoV-2 spike S1 or ACE2, we performed an in silico analysis. We applied a rigid-body protein-protein interaction tool to model the interaction between ACE2 and receptor-binding domain (RBD) of spike $\mathrm{S} 1$ in the absence or presence of eugenol. As expected, in the absence of eugenol, various residues (Lys417, Tyr449, Gly496, Asn501, and Tyr505) of spike S1 interacted with Asp30, Asp38, Gln42, and Lys353 residues of ACE2 (Fig. 1B). However, eugenol showed association with spike S1, not ACE2 (Fig. 1C). Interestingly, eugenol interacted with Lys417 residue of spike $\mathrm{S} 1$ and due to this binding, the ionic bond (salt bridge) that bound with Asp30 of ACE2 was broken and Lys417 exhibited a different rotameric pose (Fig. 1C).

To confirm the binding of eugenol with spike S1, not ACE2, we employed thermal shift assay, which is an important tool for analyzing ligand-to-receptor binding. The melting profile of full-length spike S1 and human ACE2 was monitored with the aid of a SYBR Green reaction at 27-94 ${ }^{\circ} \mathrm{C}$. Typical sigmoidal melting curves clearly indicated that recombinant spike S1 (Fig. 1D) and ACE2 (Fig. 1E) were conformationally stable. Interestingly, the melting assay revealed that $10 \mu \mathrm{M}$ of eugenol was capable of strongly shifting the melting curve of spike $\mathrm{S} 1$ by $6^{\circ} \mathrm{C}$ from $72.71{ }^{\circ} \mathrm{C}$ to $78.71{ }^{\circ} \mathrm{C}$ (Fig. 1D). In contrast, eugenol exhibited a thermal shift of only $0.7{ }^{\circ} \mathrm{C}$ with human ACE2 protein from 61.5 to $62.2{ }^{\circ} \mathrm{C}$ (Fig. 1E). In order to understand whether the complex of spike S1 and ACE2 was conformationally stable and whether eugenol was capable of influencing the preexisting complex, we examined the melting profile of the combination of spike S1 and ACE2 in the absence and presence of eugenol. As evident from sigmoidal melting curve, the complex of spike S1 and ACE2 was conformationally stable (Fig. 1F). However, the presence of eugenol led to a shift of the melting curve of the spike S1:ACE2 complex by $3.27^{\circ} \mathrm{C}$ from $59.66^{\circ} \mathrm{C}$ to $62.93^{\circ} \mathrm{C}$ (Fig. 1F). Together, these results indicate that eugenol binds to SARS-CoV-2 spike S1, but not ACE2, and that eugenol is also capable of associating with the established spike S1:ACE2 complex.

Does Eugenol Inhibit Viral Entry? Since eugenol suppressed the binding of SARS-CoV-2 spike S1 with ACE2, next, we investigated whether eugenol inhibited viral entry. Pseudoviruses are suitable for virus entry assays, as they permit viral entry to be distinguished from other virus life-cycle stages. Therefore, we used lentiviral particles pseudotyped with the SARS-CoV-2 Spike S1 protein. Since human embryonic kidney 293 (HEK293) cells do not have any detectable ACE2 receptors, we used HEK293 cells expressing human ACE2 for entry assay. In pseudovirus luciferase assay, viral entry into cells correlates to the levels of luciferase signals in the cells. While lenti-naked infection did not increase luciferase signals in hACE2-expressing HEK293 cells, marked increase in luciferase activity was seen in pseudoSARS-CoV-2-infected cells (Fig. 2A), indicating the entry of pseudo-SARS-CoV-2 into hACE2-HEK293 cells. However, eugenol at 5, 10 and $20 \mu \mathrm{M}$ concentrations strongly 
Fig. 1 Eugenol is capable of inhibiting the interaction between ACE2 and SARSCoV-2 spike $\mathrm{S} 1$. A) Chemiluminescence assay indicated inhibition of ACE2 to SARS-CoV-2 spike $\mathrm{S} 1$ interaction by different doses of eugenol, but not ursolic acid, oleanolic acid and $\beta$-caryophylline (other major components of Tulsi leaf). ***p $<0.001 ;$ NS, not significant. B) A rigid-body in silico docked pose of human ACE2 (dark yellow epoxy color) and SARSCoV-2 spike $\mathrm{S} 1$ (dark magenta) in the absence of eugenol. C) A rigid-body in silico docked pose of human ACE2 (magenta) and SARS-CoV-2 spike S1 (green) in the presence of eugenol (dark yellow structure). D) Thermal shift assay of spike $\mathrm{S} 1$ was conducted with $10 \mu \mathrm{M}$ eugenol. The melting of spike S1 was monitored using a SYBR Green real-time melting strategy. E) Thermal shift assay of human ACE2 was conducted with 10 $\mu \mathrm{M}$ eugenol. F) Thermal shift assay of the combination of spike S1 and human ACE2 was conducted with $10 \mu \mathrm{M}$ eugenol. Results were analyzed and confirmed after three independent experiments
A
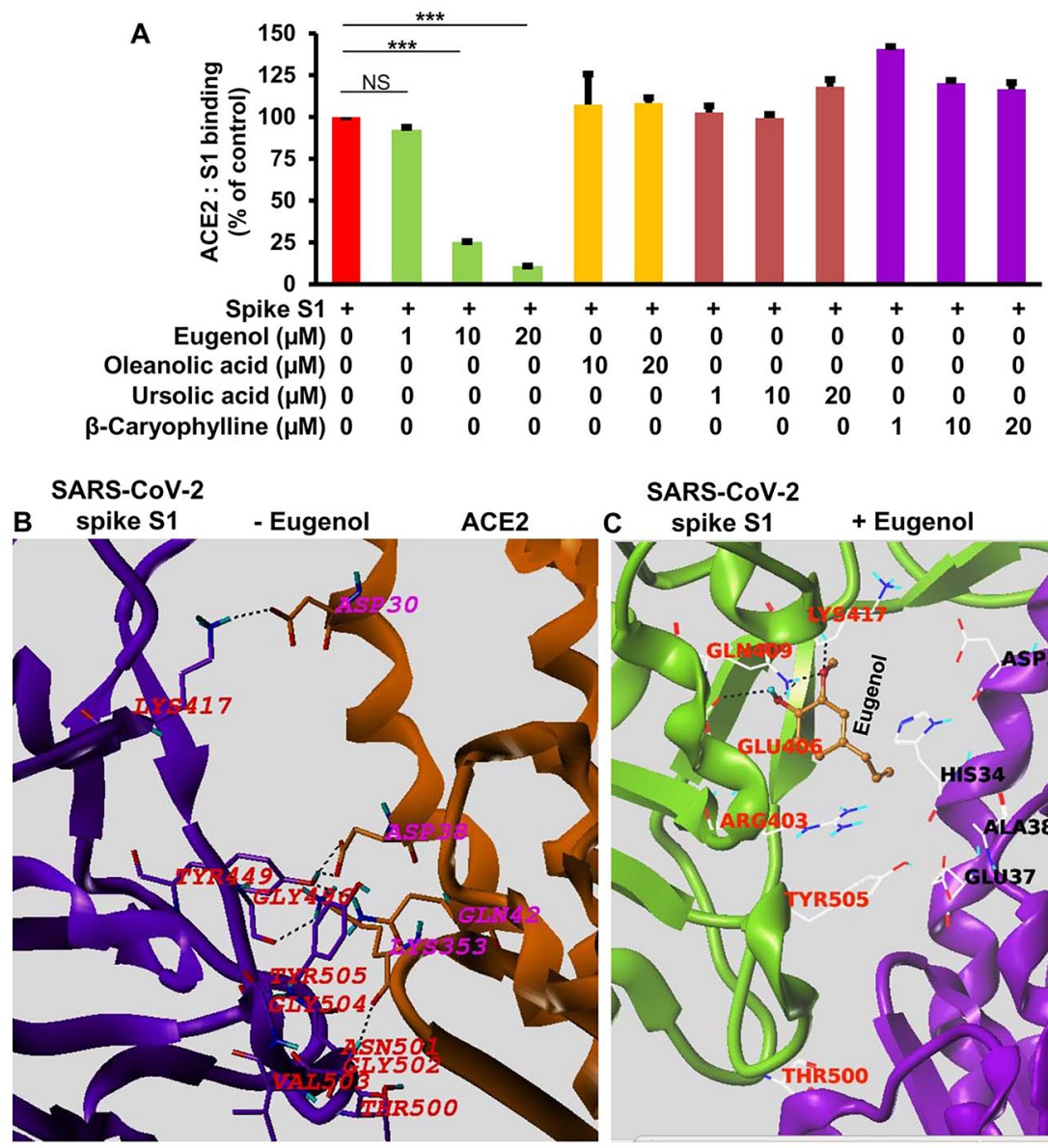

SARS-CoV-2
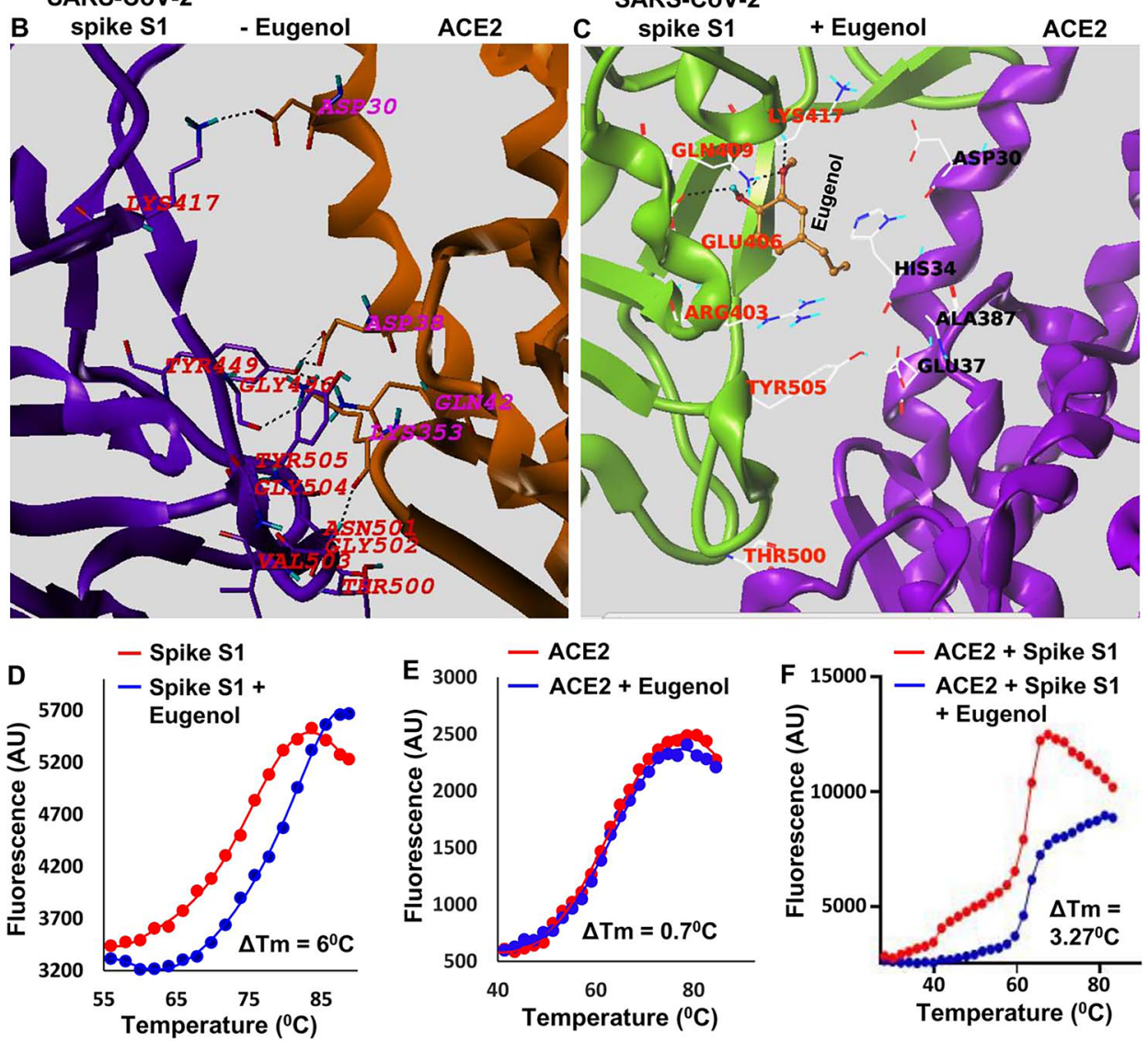

inhibited pseudo-SARS-CoV-2-induced luciferase activity (Fig. 2A), suggesting that eugenol inhibits the entry of pseudo-SARS-CoV-2 into hACE2-HEK293 cells. Similar to pseudo-SARS-CoV-2, infection with pseudo-VSV also led to marked increase in luciferase activity in hACE2HEK293 cells (Fig. 2B). However, in contrast to that seen with pseudo-SARS-CoV-2, eugenol did not inhibit pseudoVSV-induced luciferase activity in hACE2-HEK293 cells (Fig. 2B).

To further confirm eugenol-mediated inhibition of viral entry, we checked GFP fluorescence as a measure of infection. Marked GFP expression was found in cells infected with both SARS-CoV-2 pseudovirus (Fig. 2C) and VSV pseudovirus (Fig. 2D) while we did not observe GFP expression in the cells infected with lenti-naked viral particles (Fig. 2C, D). However, eugenol strongly inhibited GFP expression induced by pseudo-SARS-CoV-2 (Fig. 2C, E). On the other hand, eugenol had no effect on VSV pseudovirus-induced GFP expression in hACE2-HEK293 cells (Fig. 2D, F). Together, these results suggest that eugenol is capable of inhibiting the entry of pseudo-SARS-CoV-2, but not pseudo-VSV, into hACE2-HEK293 cells. 
Fig. 2 Effect of eugenol on the entry of pseudotyped SARSCoV-2 and VSV into hACE2expressing HEK293 cells. Cells plated at $70-80 \%$ confluence in 24-well plates were treated with eugenol for 10 min followed by infection with lentiviral particles pseudotyped with either SARS-CoV-2 spike S1 (A) or VSV-G (B) at an MOI of 0.5. After $2 \mathrm{~d}$ of infection, the entry of pseudovirus was monitored by luciferase activity in total cell extracts using the Luciferase Assay System (Promega) in a TD-20/20 Luminometer (Turner Designs, Sunnyvale, CA). Results are mean \pm SD of three independent experiments. $* * * p<0.001$. NS, not significant. After $2 \mathrm{~d}$ of infection, cells were fixed and GFP fluorescence was recorded in an Olympus BX-41 fluorescence microscope (C, SARS-CoV-2 spike S1; D, VSV-G). Results represent three independent experiments. GFP-positive cells were counted in 10 different fields of each of different treatment groups and expressed as mean GFP-positive cells per field (E, SARS-CoV-2 spike $\mathrm{S} 1$; $\mathbf{F}, \mathrm{VSV}-\mathrm{G}) .{ }^{* * *} p<0.001$. NS, not significant
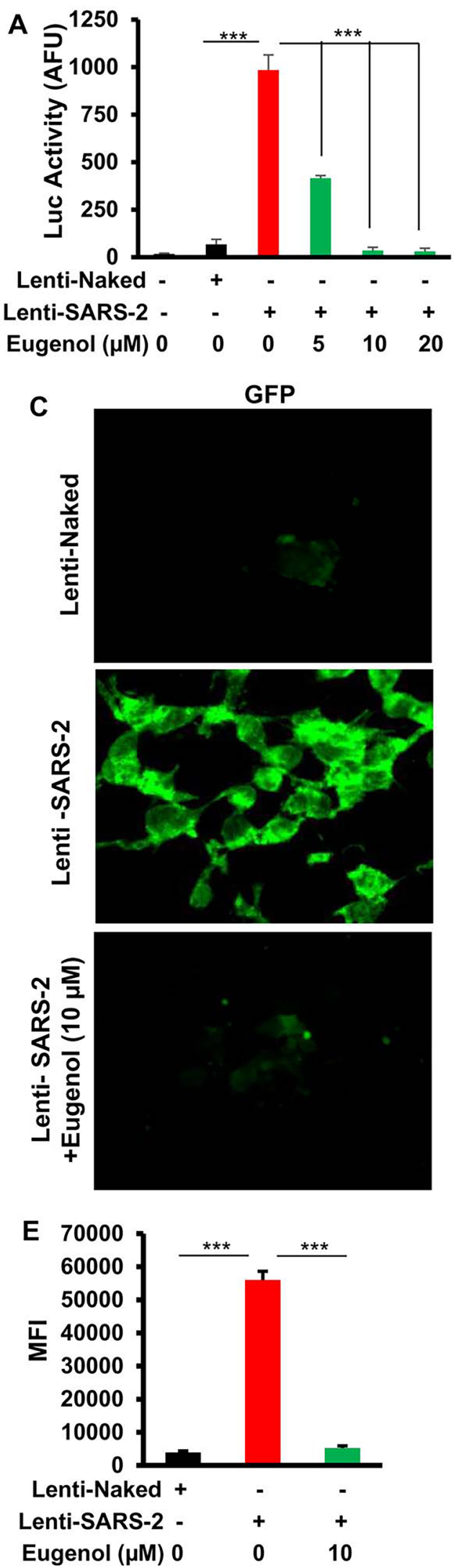
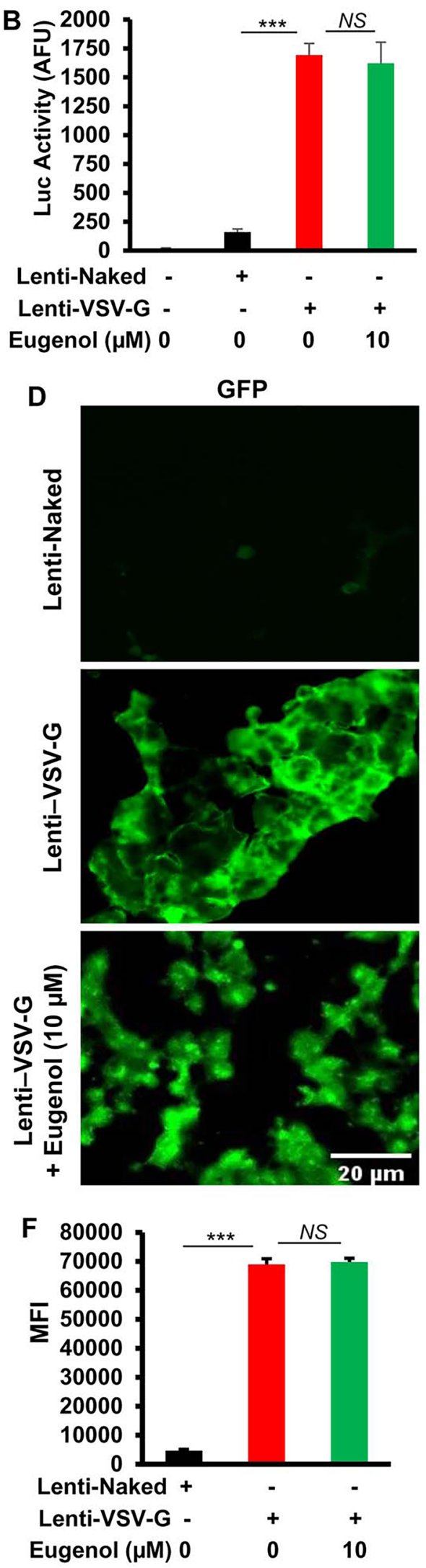
Eugenol Inhibits the Activation of NF- $\kappa$ B and the Expression of Proinflammatory Molecules in SARS-CoV-2 Spike S1-stimulated A549 Lung Cell Line Some COVID-19 patients present a severe symptom of acute respiratory distress syndrome (ARDS) with high mortality. This high severity is dependent on pulmonary inflammation induced by a cytokine storm (Pia 2020), which is most likely mediated by interleukin-6 (IL-6) and other proinflammatory cytokines. NF- $\kappa \mathrm{B}$ is a proinflammatory transcription factor (Vallabhapurapu and Karin 2009) and recently we have demonstrated that recombinant SARS-CoV-2 spike $\mathrm{S} 1$ induces the activation of NF- $\kappa$ B and the expression of IL- 6 in human A549 lung cells (Paidi et al. 2021). While heat-denatured spike $\mathrm{S} 1$ does not induce the expression of proinflammatory molecules in A549 cells, anti-spike S1 neutralizing antibody (BioVision; Cat\# A3000-50) abrogates the proinflammatory function of recombinant SARS-CoV-2 spike S1 (Paidi et al. 2021). Therefore, we examined if eugenol was capable of suppressing inflammation in human A549 lung cells induced by recombinant SARS-CoV-2 spike S1. As evident by EMSA, recombinant spike $\mathrm{S} 1$ induced the DNA-binding activity of NF- $\kappa$ B in A549 cells (Fig. 3A). However, eugenol inhibited spike S1-induced activation of NF- $\kappa$ B (Fig. 3A). To confirm these results, we monitored the expression of TNFa, IL- $1 \beta$ and IL-6, proinflammatory cytokines that are driven by activated NF- $\kappa$ B. Spike S1 increased the expression of TNFa (Fig. 3B), IL-1 $\beta$ (Fig. 3C) and IL-6 (Fig. 3D) in A549 cells. However, eugenol dose-dependently inhibited SARS-CoV-2 spike S1-induced mRNA expression of TNFa (Fig. 3B), IL-1 $\beta$ (Fig. 3C) and IL-6 (Fig. 3D) in A549 cells.

\section{Oral Administration of Eugenol Inhibits Lung Inflamma-} tion and Reduces Fever in SARS-CoV-2 Spike S1-intoxicated Mice Although SARS-CoV-2 does not easily bind to ACE2 and infect normal mice, we have observed that intranasal intoxication of SARS-CoV-2 spike S1 induces fever and important cardiac and respiratory symptoms of COVID-19 in normal C57/BL6 mice (Paidi et al. 2021). Therefore, we studied whether eugenol could reduce these symptoms in mice. Since COVID-19 patients are and/or will be treated with drugs after diagnosis of the disease, we examined whether eugenol administered $5 \mathrm{~d}$ after initiation of the disease (Fig. 4A) was still capable of defending mice from COVID-19 related complications. We selected the 5-day window as all SARS-CoV-2 spike S1-intoxicated mice exhibited a body temperature of around $100^{\circ} \mathrm{F}$ on $5 \mathrm{~d}$ of intoxication (Fig. 4B). Parallel to that observed in human lung cells, intranasal exposure with recombinant SARSCoV-2 spike S1 (Fig. 4A) led to the expression of TNFa (Fig. 4C), IL-1 $\beta$ (Fig. 4D) and IL-6 (Fig. 4E) in vivo in the lung of C57/BL6 mice. However, oral administration of eugenol strongly inhibited the mRNA expression of TNFa (Fig. 4C), IL-1 $\beta$ (Fig. 4D) and IL-6 (Fig. 4E) in the lungs

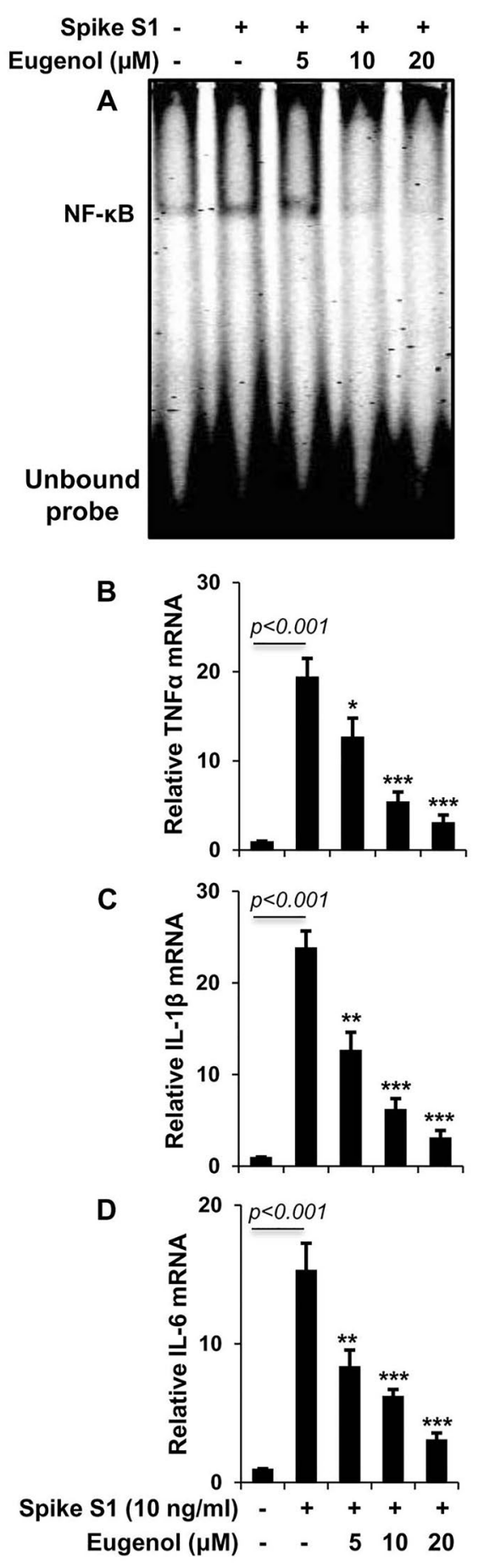

Fig. 3 Effect of eugenol on the induction of NF- $\mathrm{BB}$ activation and the expression of proinflammatory molecules in A549 lung cell line. A549 cells preincubated with eugenol for 10 min were stimulated with $10 \mathrm{ng} / \mathrm{ml}$ recombinant SARS-CoV-2 spike S1 under serum-free conditions. After $1 \mathrm{~h}$ of stimulation, NF- $\kappa \mathrm{B}$ activation was monitored in nuclear extracts by EMSA (A). Results represent three independent experiments. After $4 \mathrm{~h}$ of stimulation, the mRNA expression of TNFa (B), IL-1 $\beta$ (C) and IL-6 (D) was monitored by quantitative real-time PCR. Results are mean \pm SD of three independent experiments. ${ }^{*} p<0.05 ;{ }^{* *} p<0.01 ;{ }^{* * *} p<0.001$ vs. spike $\mathrm{S} 1$ 
of SARS-CoV-2 spike S1-insulted mice. Similarly, eugenol treatment also suppressed the level of TNFa in serum of SARS-CoV-2 spike S1-insulted mice (Fig. 4F). Fever is probably one of the prominent symptoms of COVID-19 (Machhi et al. 2020; Pahan and Pahan 2020) and oral administration of eugenol also led to the normalization of body temperature of SARS-CoV-2 spike S1-intoxicated mice (Fig. 4G).

\section{Oral Eugenol Improves Heart Functions in SARS-CoV-2 Spike} S1-intoxicated Mice Since many cardiac related problems of COVID-19 are modeled in SARS-CoV-2 spike S1-intoxicated mice (Paidi et al. 2021), we examined if oral eugenol was capable of improving heart functions in these mice. Noninvasive ECG demonstrated cardiac arrhythmias in SARSCoV-2 spike S1-intoxicated mice as compared to control untreated mice (Fig. 5A, B). However, eugenol treatment normalized electrical activity of the heart as evident from ECG (Fig. 5A-C). Similarly, eugenol also steadied heart rate (Fig. 5D), heart rate variability (Fig. 5E), JT interval (Fig. 5F), ORS interval (Fig. 5G), QT interval (Fig. 5H), and RR interval (Fig. 5I) in SARS-CoV-2 spike S1-intoxicated mice. As expected, the level of LDH was also markedly higher in serum of SARS-CoV-2 spike S1-intoxicated mice than normal
Fig. 5 Eugenol protects heart functions in a mouse model of COVID19. Eight-ten week old C57/BL6 mice $(n=7)$ of both sexes were intoxicated with recombinant SARS-CoV-2 spike S1 (50 ng/mouse/d) via intranasal route. After $5 \mathrm{~d}$ of insult, when all mice displayed a body temperature of around $100^{\circ} \mathrm{F}$, mice were treated orally with eugenol $(25 \mathrm{mg} / \mathrm{kg}$ body weight/d) via gavage. After $10 \mathrm{~d}$ of treatment, heart functions were monitored by non-invasive electrocardiography (ECG) using the PowerLab (ADInstruments) [A, chromatogram of control mice; $\mathbf{B}$, chromatogram of spike S1-intoxicated mice; C, chromatogram of (spike S1 + eugenol)-treated mice; D, heart rate; $\mathrm{E}$, heart rate variability; F, JT interval; $\mathbf{G}$, QRS interval; $\mathbf{H}, \mathrm{QT}$ interval; I, RR interval]. J) Serum LDH was quantified using an assay kit from Sigma. Results are mean \pm SEM of 7 mice per group. ${ }^{*} p<0.05$; ${ }^{* *} p<0.01 ;{ }^{* * *} p<0.001$

mice (Fig. 5J). However, eugenol treatment normalized serum LDH in spike S1-intoxicated mice (Fig. 5J).

Oral Administration of Eugenol Increases Locomotor Performance in SARS-CoV-2 Spike S1-intoxicated Mice Recently, we have demonstrated that SARS-CoV-2 spike S1 insult causes functional discrepancies in C57/BL6 mice (Paidi et al. 2021). Similarly, we found a decrease in overall locomotor activities in SARS-CoV-2 spike S1-intoxicated mice (Fig. 6A-G). Therefore, we investigated whether oral eugenol could improve such behavioral deficits. Interestingly,
Fig. 4 Oral eugenol decreases inflammation and reduces fever in a mouse model of COVID19. Eight-ten week old C57/ BL6 mice $(n=7)$ of both sexes were intoxicated with recombinant SARS-CoV-2 spike S1 (50 $\mathrm{ng} / \mathrm{mouse} / \mathrm{d}$ ) via intranasal route (A, Schematic presentation). After $5 \mathrm{~d}$ of insult, when all mice displayed a body temperature of around $100^{\circ} \mathrm{F}(\mathbf{B})$, mice were treated orally with eugenol ( $25 \mathrm{mg} / \mathrm{kg}$ body weight/d) via gavage. After $10 \mathrm{~d}$ of eugenol treatment, the mRNA expression of TNFa (C), IL-1 $\beta$ (D) and IL-6 (E) was monitored in lung by real-time PCR. Level of TNFa (F) was quantified in serum by ELISA. Body temperature (G) was monitored by Cardinal Health Dual Scale digital rectal thermometer. Results are mean \pm SEM of 7 mice per group. ${ }^{* * *} p<0.001$; NS, not significant

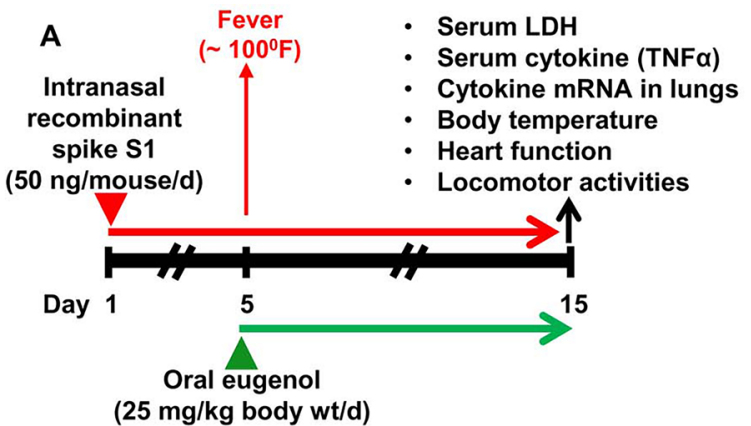

B Day 5 (Pre-eugenol)
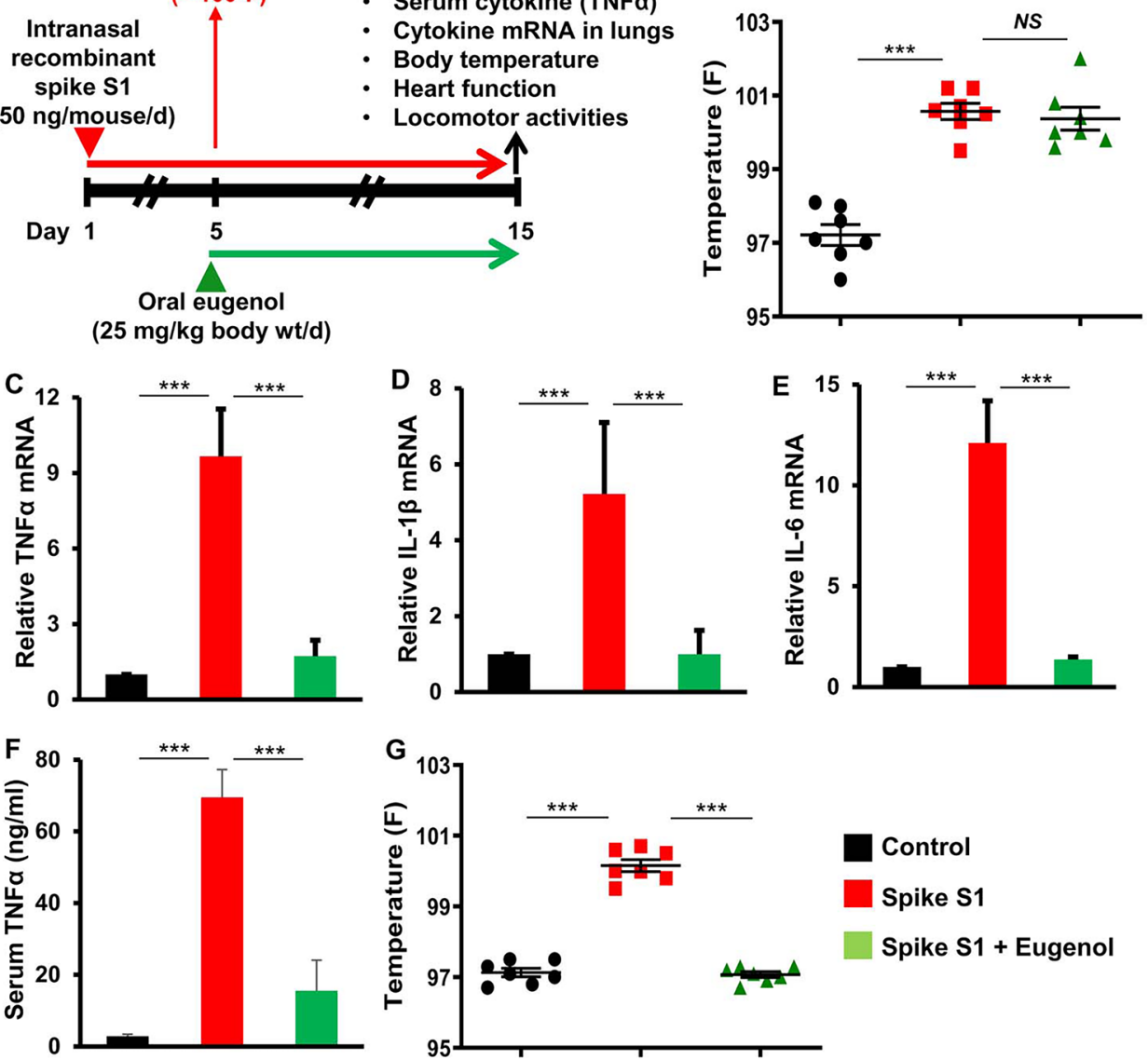


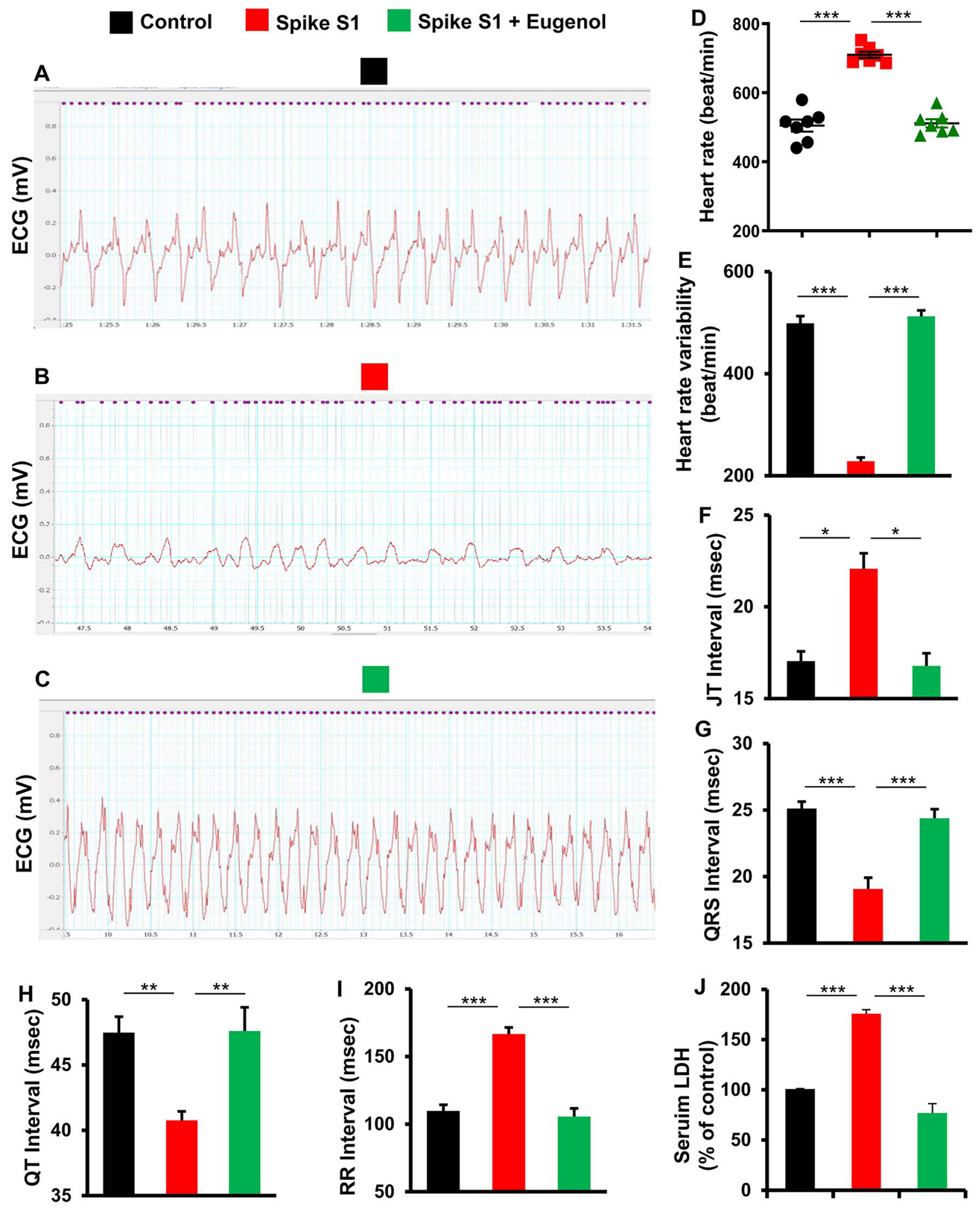

eugenol treatment increased overall locomotor activities as evident by heat map (Fig. 6A), distance travelled (Fig. 6B), velocity (Fig. 6C), cumulative duration (Fig. 6D), center zone frequency (Fig. 6E), center zone cumulative duration (Fig. 6F), and rotorod performance (Fig. 6G). We did not notice any drug-related side effect (e.g. hair loss, appetite 
loss, weight loss, untoward infection, irritation, etc.) in any mouse upon treatment with oral eugenol at a dose of $25 \mathrm{mg} /$ $\mathrm{kg}$ body weight/d.

\section{Discussion}

Although more than half of the population in the United States of America have been fully vaccinated against COVID-19, we do not know whether vaccination would be a semi-annual, annual, or biannual event. Since vaccine-induced immunity to the virus declines, the CDC has recommended that people with moderately to severely compromised immune systems receive the third dose of mRNA COVID-19 vaccine. Pfizer has proposed the third shot for everybody 6 months after receiving the second dose! This means people must continually get COVID19 vaccinations at designated time intervals. On the other hand, distribution of COVID-19 vaccines globally will take months and possibly years in certain parts of the world. Moreover, storage of some of the widely-used COVID-19 vaccines at $-40^{\circ} \mathrm{C}$ to $-80^{\circ} \mathrm{C}$ throughout the world is a major concern. We must remember that despite flu vaccination, approximately 40,000 to 50,000 people die annually from the flu in United States. Therefore, vaccines may not entirely prevent the spread of COVID-19 and it is necessary to find an effective, economical, and safe drug to better manage COVID-19 in the post-vaccine era. Since medieval times, Tulsi leaf has been being used to stimulate immunity and treat different human disorders (Prakash and Gupta 2005; Baliga et al. 2013; Cohen 2014; Jamshidi and Cohen 2017). This study underlines the importance of eugenol, a component of Tulsi leaf, in inhibiting the interaction between spike S1 and ACE2. Specificity is highlighted by our results showing ursolic acid, oleanolic acid and $\beta$-caryophylline (other major components of Tulsi leaf) remain unable to hinder the interaction between spike $\mathrm{S} 1$ and ACE2. Accordingly, in a pseudovirus cell entry assay, eugenol inhibited the entry of SARS-CoV-2 pseudovirus into hACE2-expressing HEK293 cells. In contrast, eugenol could not inhibit the entry of VSV pseudovirus into hACE2-expressing HEK293 cells, indicating the functional specificity of eugenol. Eugenol is a bioactive compound (Marchese et al. 2017; Barboza et al. 2018) and in addition to Tulsi, it is widely available in many herbs like clove, cinnamon, pepper, etc. Therefore, our results suggest that this naturally available compound may be beneficial for COVID-19.

Angiotensin-converting enzyme 2 (ACE2) being the main effector of the classical renin-angiotensin system is an important molecule for the regulation of blood pressure and hypertension as the prototype function of ACE2 is to convert angiotensin II (AngII), a vasoconstrictor, to
Ang1-7, a vasodilator (Vickers et al. 2002; Zaman et al. 2002). However, SARS-CoV-2 requires ACE2 to enter human cells. Since ACE2 is predominant in lung, heart and kidney (Zaman et al. 2002), SARS-CoV-2 easily infects these organs causing multi-organ failure in severe COVID19 cases. Therefore, inhibition of ACE2 should reduce the SARS-CoV-2 infection and associated inflammation. However, in this case, inhibition of ACE2 should not be a valid therapeutic option for COVID-19 as it is a beneficial molecule. Therefore, to target COVID-19 from a therapeutic approach, here, we focused on identifying a molecule that will bind to SARS-CoV-2 spike S1, not ACE2, in order to inhibit the interaction between spike S1 and ACE2. Interestingly, our chemiluminescence-based binding assay, in silico structural analysis and thermal shift assay clearly delineated that eugenol binds to spike $\mathrm{S} 1$, but not ACE2, to inhibit the association between ACE2 and spike $\mathrm{S} 1$. Therefore, in this case, without affecting ACE2 function, eugenol should not pose any risk for COVID-19 patients with preexisting cardiovascular, pulmonary and kidney problems. Recently, in a review, Vicidomini et al. (Vicidomini et al. 2021) have described the potential role of clove (a rich source of eugenol) in the frame of anti-COVID-19 therapy, focusing on the antiviral, anti-inflammatory, and antithrombotic effects of clove. However, it does not discuss whether and how eugenol may inhibit SARS-CoV-2 binding to its receptor and hence viral entry into host cells.

Inflammation plays a key role in the pathogenesis of many human disorders, including COVID-19. It has been shown that COVID-19 patients with severe symptoms suffer from cytokine storm (Mehta et al. 2020). We have also seen that spike $\mathrm{S} 1$ alone is capable of driving the expression of proinflammatory cytokines in human A549 lung cells (Paidi et al. 2021). Therefore, spike $\mathrm{S} 1$ component of SARS-CoV-2 may be responsible for cytokine storm seen in COVID-19 patients. Gene promoters of most of the proinflammatory molecules harbor binding site(s) for NF- $\mathrm{kB}$ (Saha and Pahan 2006; Lawrence 2009; Hayden and Ghosh 2014). Accordingly, activation of NF- $\kappa B$ plays an essential role in the transcription of most of the proinflammatory molecules (Saha and Pahan 2006; Lawrence 2009). Consistent to the inhibition of binding between ACE2 and SARS-CoV-2 spike S1, eugenol decreased the activation of NF- $\kappa B$ and the mRNA expression of TNFa, IL- $1 \beta$ and IL-6 in SARS-CoV-2 spike S1-challenged A549 lung cells. Therefore, eugenol may be able to take care of inflammation associated to COVID-19.

Several studies have described antioxidant potential of eugenol (Gulcin 2011; Barboza et al. 2018). It has been shown that the antioxidant behavior of eugenol is greater than most of the known or standard antioxidants such as Trolox (Gulcin 2011). According to Jin and Cho (Jin and Cho 2011), eugenol decreases lipidemia in hyperlipidemic 
Fig. 6 Oral administration of eugenol improves locomotor activities in a mouse model of COVID-19. Eight-ten week old C57/BL6 mice $(n=7)$ of both sexes were intoxicated with recombinant SARS-CoV-2 spike $\mathrm{S} 1$ (50 ng/mouse/d) via intranasal route. After $5 \mathrm{~d}$ of insult, when all mice displayed a body temperature of around $100^{0} \mathrm{~F}$, mice were treated orally with eugenol ( $25 \mathrm{mg} / \mathrm{kg}$ body weight/d) via gavage. After 10 $\mathrm{d}$ of treatment, mice were tested for general locomotor activities (A, heat map; $\mathbf{B}$, distance travelled; C, velocity; D, cumulative duration; $\mathbf{E}$, center zone frequency; $\mathbf{F}$, center zone cumulative duration; $\mathbf{G}$, rotorod latency). Results are mean \pm SEM of 7 mice per group. ${ }^{*} p<$ $0.05 ;{ }^{* *} p<0.01 ;{ }^{* * * *} p<0.001$
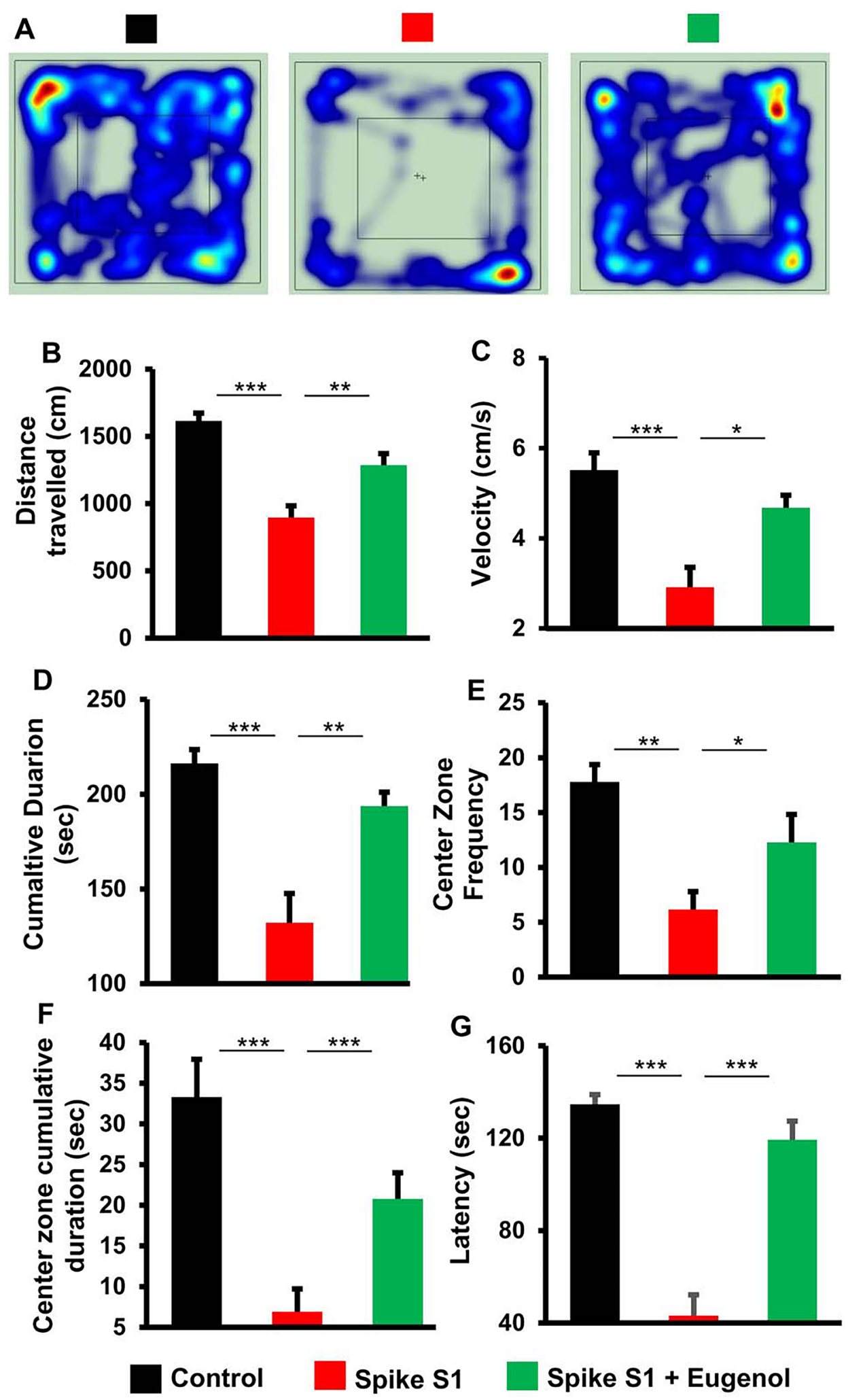

zebra fish model, where eugenol causes a great reduction in triglyceride and cholesterol levels in serum samples. Diabetes is an important risk factor for COVID-19 (Kumar et al. 2020) and eugenol has been shown to enhance the activities of carbohydrate metabolism enzymes such as glucose-6-phosphate dehydrogenase and instance hexokinase (Srinivasan et al. 2014). Moreover, in a number of studies (Jaganathan and Supriyanto 2012; Fujisawa and Murakami 
2016), eugenol has shown efficacy against a number of diseases such as reproductive disorders, nervous system disorders, microbial infections, tumorigenesis, hypertension, inflammations, and digestive complications. Here, we delineate a new property of eugenol in inhibiting the binding of SARS-CoV-2 spike S1 with ACE2 and suppressing viral entry into host cells. Reduction of lung inflammation, normalization of heart functions, reduction of fever, decrease in serum markers, and improvement in locomotor activities in SARS-CoV-2 spike S1-intoxicated mice by oral administration of eugenol suggest that oral eugenol may be beneficial for COVID-19. Although COVID-19 is the first documented coronavirus pandemic in the history (Liu et al. 2020), we must remember that several human-infecting coronaviruses are there with distinctive degrees of virulence. Since the first step of coronavirus pathogenesis is the attachment of coronavirus to the host via viral spike glycoprotein, in addition to COVID-19, oral eugenol may be effective against other coronaviruses.

Acknowledgements This study was supported by grants (AG050431, AG069229 and AT010980) from NIH to KP. Moreover, KP is the recipient of a Research Career Scientist Award (1IK6 BX004982) from the Department of Veterans Affairs.

\section{Declarations}

Conflict of Interest None.

\section{References}

Baliga MS, Jimmy R, Thilakchand KR, Sunitha V, Bhat NR, Saldanha E, Rao S, Rao P, Arora R, Palatty PL (2013) Ocimum sanctum L (Holy Basil or Tulsi) and its phytochemicals in the prevention and treatment of cancer. Nutr Cancer 65(Suppl 1):26-35

Barboza JN, da Silva Maia Bezerra Filho C, Silva RO, Medeiros JVR, de Sousa DP (2018) An Overview on the Anti-inflammatory Potential and Antioxidant Profile of Eugenol. Oxid Med Cell Longev 2018:3957262

Cantuti-Castelvetri L et al (2020) Neuropilin-1 facilitates SARS-CoV-2 cell entry and infectivity. Science 370:856-860

Cohen MM (2014) Tulsi - Ocimum sanctum: A herb for all reasons. J Ayurveda Integr Med 5:251-259

Du L, He Y, Zhou Y, Liu S, Zheng BJ, Jiang S (2009) The spike protein of SARS-CoV--a target for vaccine and therapeutic development. Nat Rev Microbiol 7:226-236

Fujisawa S, Murakami Y (2016) Eugenol and Its Role in Chronic Diseases. Adv Exp Med Biol 929:45-66

Gulcin I (2011) Antioxidant activity of eugenol: a structure-activity relationship study. J Med Food 14:975-985

Hayden MS, Ghosh S (2014) Regulation of NF-kappaB by TNF family cytokines. Semin Immunol 26:253-266

Jaganathan SK, Supriyanto E (2012) Antiproliferative and molecular mechanism of eugenol-induced apoptosis in cancer cells. Molecules 17:6290-6304

Jamshidi N, Cohen MM (2017) The Clinical Efficacy and Safety of Tulsi in Humans: A Systematic Review of the Literature. Evid Based Complement Alternat Med 2017:9217567
Jana M, Palencia CA, Pahan K (2008) Fibrillar amyloid-beta peptides activate microglia via TLR2: implications for Alzheimer's disease. J Immunol 181:7254-7262

Jana A, Modi KK, Roy A, Anderson JA, van Breemen RB, Pahan K (2013) Up-regulation of neurotrophic factors by cinnamon and its metabolite sodium benzoate: therapeutic implications for neurodegenerative disorders. J Neuroimmune Pharmacol 8:739-755

Jin S, Cho KH (2011) Water extracts of cinnamon and clove exhibits potent inhibition of protein glycation and anti-atherosclerotic activity in vitro and in vivo hypolipidemic activity in zebrafish. Food Chem Toxicol 49:1521-1529

Kumar A, Arora A, Sharma P, Anikhindi SA, Bansal N, Singla V, Khare S, Srivastava A (2020) Is diabetes mellitus associated with mortality and severity of COVID-19? A meta-analysis. Diabetes Metab Syndr 14:535-545

Lawrence T (2009) The nuclear factor NF-kappaB pathway in inflammation. Cold Spring Harb Perspect Biol 1:a001651

Ledford H (2020) How does COVID-19 kill? Uncertainty is hampering doctors' ability to choose treatments. Nature 580:311-312

Liu YC, Kuo RL, Shih SR (2020) COVID-19: The first documented coronavirus pandemic in history. Biomed $\mathrm{J} 43: 328-333$

Machhi J, Herskovitz J, Senan AM, Dutta D, Nath B, Oleynikov MD, Blomberg WR, Meigs DD, Hasan M, Patel M, Kline P, Chang RC, Chang L, Gendelman HE, Kevadiya BD (2020) The Natural History, Pathobiology, and Clinical Manifestations of SARS-CoV-2 Infections. J Neuroimmune Pharmacol 15:359-386

Marchese A, Barbieri R, Coppo E, Orhan IE, Daglia M, Nabavi SF, Izadi M, Abdollahi M, Nabavi SM, Ajami M (2017) Antimicrobial activity of eugenol and essential oils containing eugenol: A mechanistic viewpoint. Crit Rev Microbiol 43:668-689

Mehta P, McAuley DF, Brown M, Sanchez E, Tattersall RS, Manson JJ, Hlh Across Speciality Collaboration UK (2020) COVID-19: consider cytokine storm syndromes and immunosuppression. Lancet 395:1033-1034

Mondal S, Varma S, Bamola VD, Naik SN, Mirdha BR, Padhi MM, Mehta N, Mahapatra SC (2011) Double-blinded randomized controlled trial for immunomodulatory effects of Tulsi (Ocimum sanctum Linn.) leaf extract on healthy volunteers. J Ethnopharmacol 136:452-456

Mondal S, Kundu M, Jana M, Roy A, Rangasamy SB, Modi KK, Wallace J, Albalawi YA, Balabanov R, Pahan K (2020) IL-12 p40 monomer is different from other IL-12 family members to selectively inhibit IL-12Rbeta1 internalization and suppress EAE. Proc Natl Acad Sci U S A 117:21557-21567

Mukherjee S, Pahan K (2021) Is COVID-19 Gender-sensitive?. J Neuroimmune Pharmacol 16:38-47

Pahan K, Sheikh FG, Namboodiri AM, Singh I (1997) Lovastatin and phenylacetate inhibit the induction of nitric oxide synthase and cytokines in rat primary astrocytes, microglia, and macrophages. J Clin Invest 100:2671-2679

Pahan K, Sheikh FG, Liu X, Hilger S, McKinney M, Petro TM (2001) Induction of nitric-oxide synthase and activation of NFkappaB by interleukin- 12 p40 in microglial cells. J Biol Chem 276:7899-7905

Pahan P, Pahan K (2020) Smooth or Risky Revisit of an Old Malaria Drug for COVID-19?. J Neuroimmune Pharmacol 15:174-180

Paidi RK, Jana M, Mishra RK, Dutta D, Raha S, Pahan K (2021) ACE2-interacting Domain of SARS-CoV-2 (AIDS) Peptide Suppresses Inflammation to Reduce Fever and Protect Lungs and Heart in Mice: Implications for COVID-19 Therapy. J Neuroimmune Pharmacol 16:59-70

Patel D, Roy A, Kundu M, Jana M, Luan CH, Gonzalez FJ, Pahan K (2018) Aspirin binds to PPARalpha to stimulate hippocampal plasticity and protect memory. Proc Natl Acad Sci U S A 115:E7408-E7417

Patel D, Jana A, Roy A, Pahan K (2019) Cinnamon and its Metabolite Protect the Nigrostriatum in a Mouse Model of Parkinson's 
Disease Via Astrocytic GDNF. J Neuroimmune Pharmacol 14:503-518

Pia L (2020) Spatial resolution of SARS-CoV-2 lung infection. Nat Rev Immunol

Prakash P, Gupta N (2005) Therapeutic uses of Ocimum sanctum Linn (Tulsi) with a note on eugenol and its pharmacological actions: a short review. Indian J Physiol Pharmacol 49:125-131

Rangasamy SB, Jana M, Roy A, Corbett GT, Kundu M, Chandra S, Mondal S, Dasarathi S, Mufson EJ, Mishra RK, Luan CH, Bennett DA, Pahan K (2018) Selective disruption of TLR2-MyD88 interaction inhibits inflammation and attenuates Alzheimer's pathology. J Clin Invest 128:4297-4312

Rangasamy SB, Dasarathi S, Pahan P, Jana M, Pahan K (2019) LowDose Aspirin Upregulates Tyrosine Hydroxylase and Increases Dopamine Production in Dopaminergic Neurons: Implications for Parkinson's Disease. J Neuroimmune Pharmacol 14:173-187

Roy A, Jana M, Corbett GT, Ramaswamy S, Kordower JH, Gonzalez FJ, Pahan K (2013) Regulation of cyclic AMP response element binding and hippocampal plasticity-related genes by peroxisome proliferator-activated receptor alpha. Cell Rep 4:724-737

Roy A, Jana M, Kundu M, Corbett GT, Rangaswamy SB, Mishra RK, Luan CH, Gonzalez FJ, Pahan K (2015) HMG-CoA Reductase Inhibitors Bind to PPARalpha to Upregulate Neurotrophin Expression in the Brain and Improve Memory in Mice. Cell Metab 22:253-265

Roy A, Kundu M, Jana M, Mishra RK, Yung Y, Luan CH, Gonzalez FJ, Pahan K (2016) Identification and characterization of PPARalpha ligands in the hippocampus. Nat Chem Biol 12:1075-1083

Saha RN, Pahan K (2006) Regulation of inducible nitric oxide synthase gene in glial cells. Antioxid Redox Signal 8:929-947
Saha RN, Liu X, Pahan K (2006) Up-regulation of BDNF in astrocytes by TNF-alpha: a case for the neuroprotective role of cytokine. $\mathrm{J}$ Neuroimmune Pharmacol 1:212-222

Sahoo M, Jena L, Rath SN, Kumar S (2016) Identification of Suitable Natural Inhibitor against Influenza A (H1N1) Neuraminidase Protein by Molecular Docking. Genomics Inform 14:96-103

Srinivasan S, Sathish G, Jayanthi M, Muthukumaran J, Muruganathan U, Ramachandran V (2014) Ameliorating effect of eugenol on hyperglycemia by attenuating the key enzymes of glucose metabolism in streptozotocin-induced diabetic rats. Mol Cell Biochem 385:159-168

Stower H (2020) Spread of SARS-CoV-2. Nat Med 26:465

Vallabhapurapu S, Karin M (2009) Regulation and function of NFkappaB transcription factors in the immune system. Annu Rev Immunol 27:693-733

Vicidomini C, Roviello V, Roviello GN (2021) Molecular Basis of the Therapeutical Potential of Clove (Syzygium aromaticum L.) and Clues to Its Anti-COVID-19 Utility. Molecules 26

Vickers C, Hales P, Kaushik V, Dick L, Gavin J, Tang J, Godbout K, Parsons T, Baronas E, Hsieh F, Acton S, Patane M, Nichols A, Tummino P (2002) Hydrolysis of biological peptides by human angiotensin-converting enzyme-related carboxypeptidase. J Biol Chem 277:14838-14843

Zaman MA, Oparil S, Calhoun DA (2002) Drugs targeting the reninangiotensin-aldosterone system. Nat Rev Drug Discov 1:621-636

Publisher's Note Springer Nature remains neutral with regard to jurisdictional claims in published maps and institutional affiliations. 